\title{
Implementation of the Dissection Theorem in Cadence Virtuoso
}

\author{
Jochen Verbrugghe, Bart Moeneclaey and Jan Vandewege \\ Ghent University, Department of Information Technology \\ Email: see http://www.intec.ugent.be/design/group.php
}

\begin{abstract}
This paper describes a tool for the Cadence Virtuoso software that implements the Dissection Theorem (DT) or General Network Theorem (GNT) and its applications: the Extra Element Theorem (EET), Chain Theorem (CT) and General Feedback Theorem (GFT). The tool allows a designer to gain additional circuit insight by providing all second- and third-level transfer functions of the DT. In particular, feedback networks are factored into their exact components, enabling a deeper insight into the structure of the loop gain, direct forward transmission and hence closed-loop behaviour.
\end{abstract}

\section{INTRODUCTION}

During circuit design, simulators such as Spectre are widely used to verify circuit behaviour. Rather than examining transfer functions from input to output, additional insight can be gained using several simpler transfer functions, each portraying a subset of the circuit properties. The tool for Cadence Virtuoso described in this paper incorporates the DT to provide these simpler transfer functions.

The structure of this paper is as follows. Section II gives an overview of the DT and its resulting applications. In Section III the tool as implemented in Cadence Virtuoso is described. Examples of the application of the tool are presented in Section IV. Finally, in Section V, conclusions are presented.

\section{Overview of the General Network TheOrem}

The General Network Theorem or Dissection Theorem [1] states that any transfer function $H$ (the first-level transfer function) of a linear network, whether feedback is involved or not, can be factored into three second-level transfer functions. This is done by using a single extra test signal (either voltage or current) injection:

$$
H=H_{\infty} \frac{1+\frac{1}{T_{n}}}{1+\frac{1}{T}}
$$

By injecting two extra test signals (voltage and current), $T$ and $T_{n}$ can further be factored into third-level transfer functions:

$$
\begin{aligned}
T & =\frac{T_{f w d}}{1+T_{\text {rev }}} \\
T_{f w d} & =T_{v, \text { fwd }} \| T_{i, \text { fwd }} \\
T_{\text {rev }} & =T_{v, \text { rev }} \| T_{i, \text { rev }}
\end{aligned}
$$

Similar expressions hold for $T_{n}$. Another-equivalent-form of (1) is given by:

$$
H=H_{\infty} \frac{T}{1+T}+\frac{H_{0}}{1+T}
$$

with the redundancy relation:

$$
\frac{H_{\infty}}{H_{0}}=\frac{T_{n}}{T}
$$

Various other equivalent forms exist [1], all of which are supported. For feedback systems, (5) is formally equal to the asymptotic gain model [2]. Remark that although the symbols used in (1)-(6) indicate the use of the DT in feedback systems, their position in the factorization merely reflects the method used to calculate them. Most of the secondand third-level transfer functions are calculated using null double injection (ndi) or double null triple injection (dnti) techniques [3]. These techniques use two (dni) or three (dnti) test signal injection sources, which are mutually adjusted to null a specific signal. By propagation of this null through the circuit, these calculations are always easier than normal single injection calculations. This comes in handy for symbolic hand analysis during the initial design phase. Furthermore, the results automatically come out in low-entropy form [4].

Depending on the test signal injection configuration, the DT morphs into the Chain Theorem [5], Extra Element Theorem [6] or General Feedback Theorem [1], in which the secondand/or third-level transfer functions have a particular meaning and can be interpreted in relation to the circuit elements.

\section{DESCRIPTION OF THE TOOL}

The tool consists of three parts: a probe to be inserted in the circuit, a SKILL script to post-process the simulation results and a presentation layer.

Fig. 1 depicts the probe and its properties. The probe has two terminals and a reference terminal. Terminals $x x$ and $y y$ inject and measure signals. The user a) inserts the probe in the circuit such that the test signal injection configuration can null the correct signals - corresponding to the desired interpretation of the DT, b) indicates input and output signal and c) selects the correct type in the probe properties. The reader is referred to Section IV for examples. The probe utilizes a custom netlist procedure, such that during netlisting a number of $\mathrm{AC}$ analyses are inserted in the netlist from which the final results are gathered. It is possible to specify parameters for these analyses. In addition the probe can be disabled.

The post-processing script calculates the second- and thirdlevel transfer functions using combinations of signals obtained from the aforementioned $\mathrm{AC}$ analyses, extending the method proposed in [7]. 


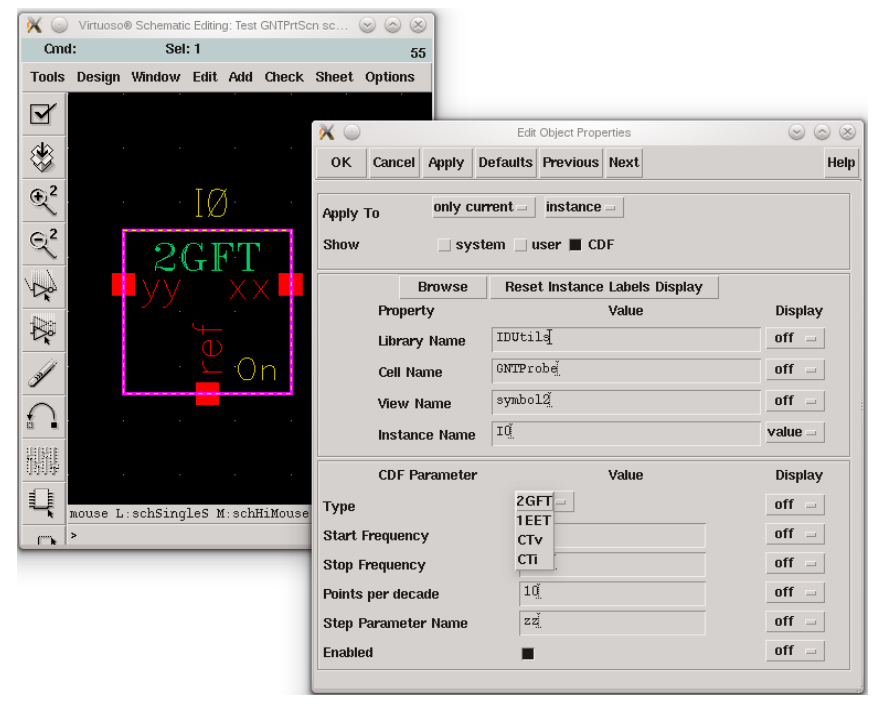

Fig. 1. DT Probe and its properties.

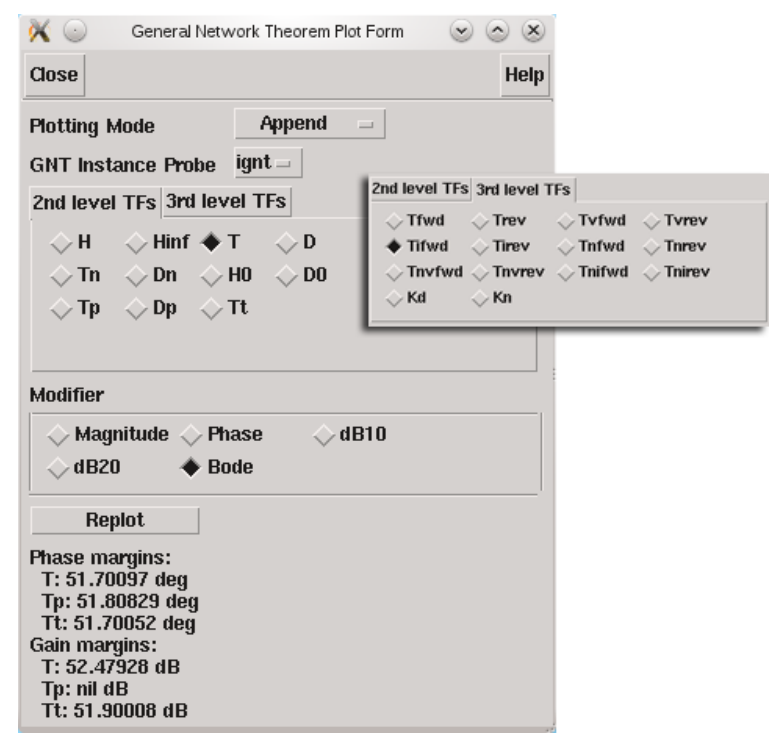

Fig. 2. DT Plot Form.

The resulting transfer functions can be accessed via a dedicated form, mimicking Cadence's own Direct Plot Form, as depicted in Fig. 2. Second- and third level transfer functions are logically split across two separate tab sheets. Additionally, stability margins are indicated for the GFT interpretation.

\section{AppliCATION EXAMPLES}

In order to illustrate the usage of the tool, an example is given for each theorem. Although no symbolic expressions are given in the examples, they can easily be calculated and compared to the numerical results. Conversely, initial simulation results can be used to find out which lower level transfer functions are dominant, as a starting point for symbolic analysis.

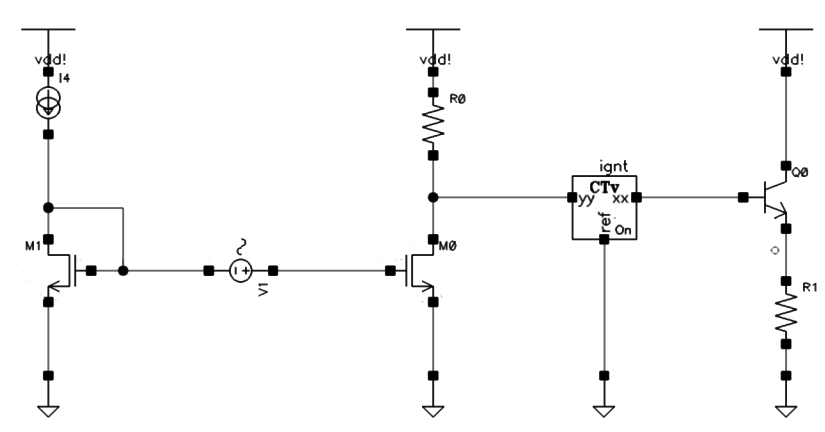

Fig. 3. CS amplifier loaded by an emitter follower to illustrate the CT.

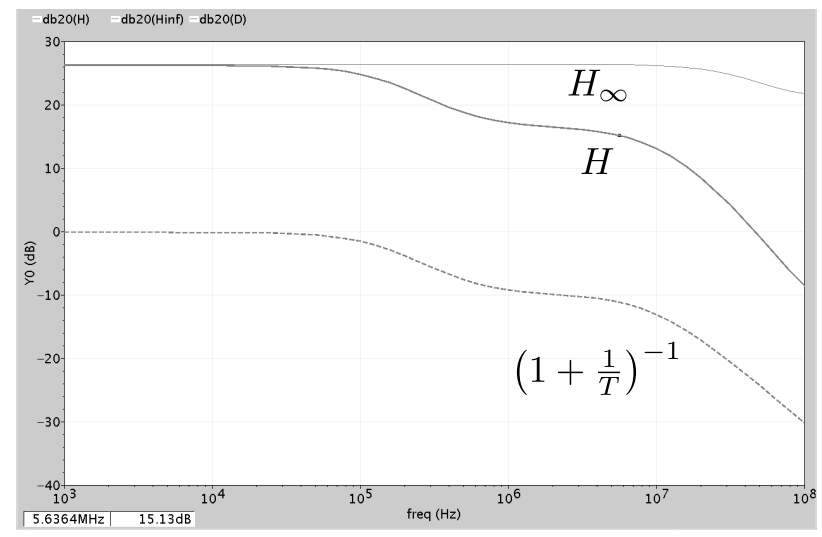

Fig. 4. Second-level transfer functions illustrating the DT as the CT.

\section{A. Chain Theorem}

The Chain Theorem is deduced from the DT with single test current or voltage injection configuration, such that the entire signal from the output of a first stage flows to the input of a second stage [5] . For voltage injection, it can be shown that $H_{\infty}$ represents the buffered voltage gain between the two stages. $T_{n}$ is infinite and $T$ equals $\frac{Z_{i 2}}{Z_{o 1}}$, the ratio of the input impedance of the second stage to the output impedance of the first stage, such that $\left(1+\frac{1}{T}\right)^{-1}$ is $\frac{Z_{i 2}}{Z_{i 2}+Z_{o 1}}$. The latter expression is a voltage division and represents the loading of the first stage by the second one. A dual form exists for current injection and current gain.

The common-source (CS) amplifier loaded by an emitter follower, shown in Fig. 3, illustrates the CT. The current mirror biases the CS stage. The input voltage is injected between the two gates, hence its Thévenin output impedance is the output impedance of the diode-connected FET. Fig. 4 shows a WaveScan screenshot of the magnitude of the resulting secondlevel transfer functions. The flat part of $H_{\infty}$ is determined by the FET transconductance, drain load resistance (first stage) and emitter follower gain (second stage), while the effect of gate-source and Miller capacitances starts to show at higher frequencies. The loading due to the emitter follower is expressed in the $\left(1+\frac{1}{T}\right)^{-1}$ factor. Note in particular the lowpass effect due to the bootstrapped base-emitter capacitance of the follower. 


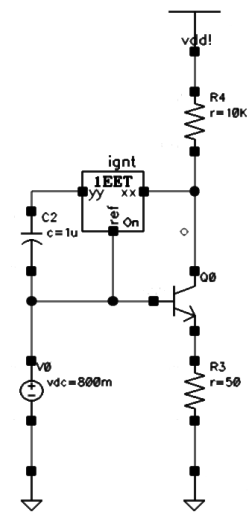

Fig. 5. Degenerated common-emitter amplifier to illustrate the Miller effect by EET.

\section{B. Extra Element Theorem}

This theorem states that any transfer function of a linear system can be expressed in terms of its value when a given extra element (EE) $Z$ is absent, and a correction factor involving the EE and two driving point impedances seen by the element [3]. Two dual versions exist, with either zero or infinite reference impedance [8]. It is deduced from the DT with single test voltage injection configuration. For the first version, the voltage injection should be placed such that the voltage across the EE, and hence the current flowing through it, can be nulled. It follows that $H_{\infty}$ then equals $\left.H\right|_{Z=\infty}$, $T$ represents the ratio of the $\mathrm{EE}$ impedance to the driving point impedance at the EE terminals $\frac{Z}{Z_{d}}$ and $T_{n}$ is the ratio of the $\mathrm{EE}$ impedance to the null driving point impedance at the EE terminals $\frac{Z}{Z_{n}}$. A similar argument holds for the the version with zero reference impedance, resulting in $H_{\infty}$ equaling $\left.H\right|_{Z=0}$ and inverted forms of $T$ and $T_{n}$. Versions of the theorem for two [6] or any number [9] of extra elements also exist.

Consider the degenerated common-emitter amplifier with explicit collector-base capacitance in Fig. 5. The EET will be used to show the Miller effect. The probe has been placed such as to obtain the EET with infinite reference impedance (shunt capacitance absent). Fig. 6 shows the second-level transfer functions. $H_{\infty}$ is flat and is mainly determined by the collector load resistance, transistor transconductance and emitter resistance. The factor $\left(1+\frac{1}{T}\right)^{-1}$ in (1) forms the lowpass filter created by the ohmic base resistance and the Miller capacitance. The well-known right half plane zero, created by both a direct forward path and an inverting path from input to output, appears in the factor $1+\frac{1}{T_{n}}$. It is mainly determined by the shunt capacitance and effective transistor transconductance. It is easily seen on the Bode diagram that the total transfer $H$ is constructed from $H_{\infty}$ and these factors: it starts and ends out flat, with a pole and zero in between.

\section{General Feedback Theorem}

The desired interpretation of the DT for feedback systems is as follows [1]: $H_{\infty}$ represents the 'ideal' transfer function

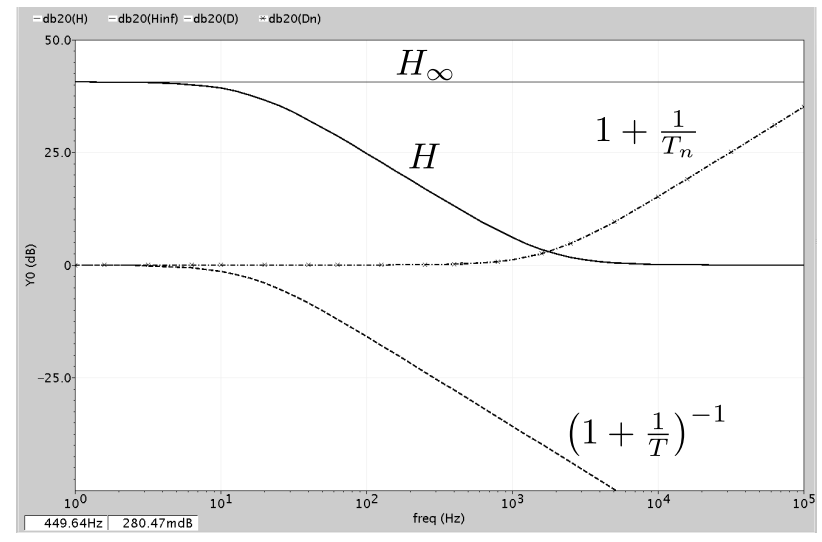

Fig. 6. Second-level transfer functions illustrating the DT as the EET.

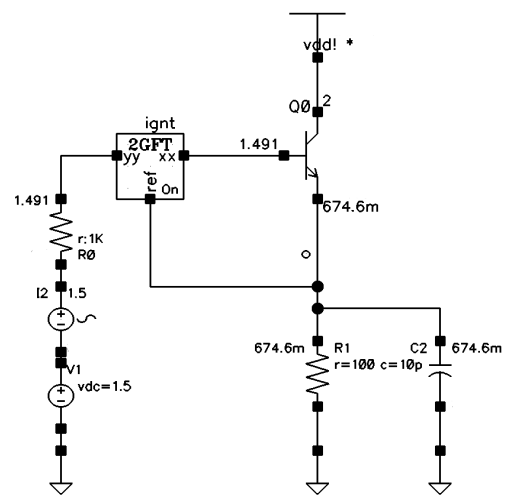

Fig. 7. Emitter follower with probe for feedback analysis.

with infinite loop gain. $T$ is the loop gain and $T_{n}$ the null loop gain. $H_{0}$ exhibits the direct forward transmission, important when the loop gain becomes small. Their true values are obtained, without any approximations or assumptions, via the DT approach, by injecting both a voltage and current such that the total feedback error signal-both voltage and current-can be nulled. Hence, the loop gain and null loop gain consist of a forward and reverse part (see (2)), both of which consist of the parallel combination of a part due to voltage injection and a part due to current injection ((3)-(4)).

As an example of local negative feedback, consider the emitter follower in Fig. 7. It is biased and driven by a $1 \mathrm{k} \Omega$ Thévenin source. The output voltage is taken from the emitter. The probe is inserted between the base and emitter, to null the error signal. A screenshot of the magnitude of the simulated second-level transfer functions is depicted in Fig. 8. As expected for a voltage buffer, $H_{\infty}$ equals $0 \mathrm{~dB}$ for all frequencies. The null loop gain $T_{n}$ is much bigger than the loop gain $T$, hence it can be safely ignored in this case. Stated otherwise, the direct forward transmission $H_{0}$ is very low. It follows that the closed-loop transfer function $H$ is determined only by $T$ and $H_{\infty}$. As seen in the figure, $T$ starts out flat around $25 \mathrm{~dB}$ and assumes a second-order slope asymptote at high frequencies. A first pole of $T$ occurs at relatively low frequency, while the second one lies close to the unity-gain 


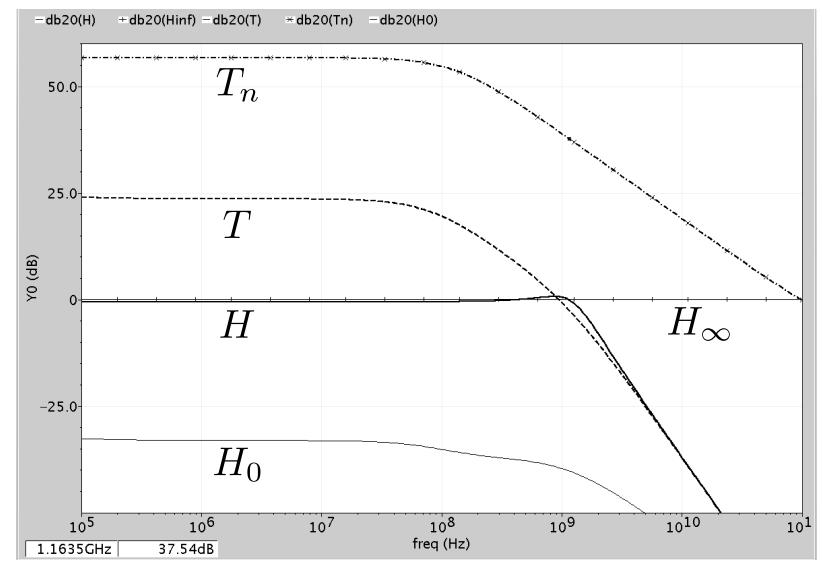

Fig. 8. Second-level transfer functions of the emitter follower illustring the DT as the GFT.

frequency. This leads to peaking in $H$, which is apparent from the plot.

Let's now conduct a more detailed analysis of the loop gain. Consider Fig. 9. As the transistor is very much unilateral, the total reverse loop gain $T_{r e v}$ is very low and can be ignored. It then follows from (2) that the loop gain equals the forward loop gain $T_{f w d}$, which is the parallel combination of a voltage injection part $T_{v, f w d}$ and a current injection part $T_{i, f w d} . T_{v, f w d}$ has a first-order behaviour and dominates at low frequencies. On the other hand, $T_{i, f w d}$ has a second-order behaviour, it has a higher DC-gain but starts falling off at lower frequencies before encountering a second pole around its unity-gain frequency. Hence, $T_{i, f w d}$ dominates at higher frequencies and consequently determines the AC-behaviour of the total closed-loop transfer function $H$. Note that the unitygain frequency of $T_{v, f w d}$ lies more than three times higher.

On a Bode diagram, a parallel operation is readily performed and the total loop gain $T$ can be constructed by just looking at the Bode diagram. We conclude that the voltage loop gain determines the low frequency gain of the emitter follower in the example, while the current loop gain determines its bandwidth. Both are required.

Comparison with Spectre's stability (stb) analysis: the stb analysis built into Spectre calculates the loop gain as Tian's loop gain, defined as [10]:

$$
T_{t}=T_{f w d}+T_{r e v}
$$

Compared with (2), both definitions become increasingly more different when the relative importance of the reverse loop gain increases, f.i. where elements cannot be considered unilateral. Moreover, the relation of $T_{t}$ to the closed-loop transfer function is not readily apparent, while that of $T$ inherently follows from the DT (1). In addition, the DT method provides all other transfer functions of interest. Note however, that both definitions satisfy for stability assertion [7], as the characteristic polynomial of the closed-loop transfer function is equal in both cases. In the given example, since $T_{r e v}$ is very low, $T_{t}$ is almost equal to $T$.

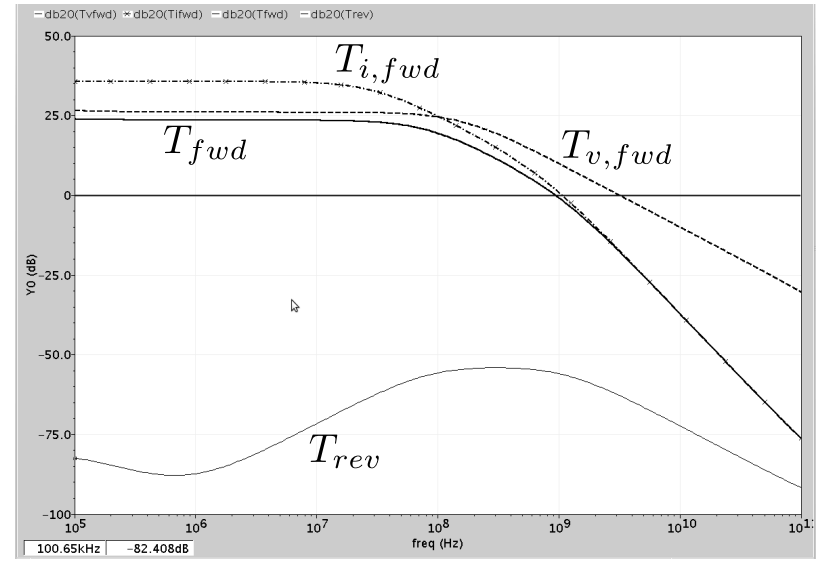

Fig. 9. Third-level transfer functions of the emitter follower, showing the components of the loop gain.

\section{CONCLUSION}

The described tool for Cadence can be of great help when trying to gain insight into the workings of a circuit. Results obtained by hand analysis using fast ndi and dnti techniques can be checked for correctness. Approximations for the hand analysis can be asserted a priori or validated a posteriori. During the design phase it can pinpoint the source of undesired circuit behaviour.

\section{ACKNOWLEDGMENT}

The authors would like to acknowledge the Agency for Innovation by Science and Technology in Flanders (IWT).

\section{REFERENCES}

[1] R. D. Middlebrook, "The general feedback theorem: A final solution for feedback systems," IEEE Microwave Magazine, vol. 7, no. 2, pp. 50+, APR 2006.

[2] P. R. Gray, P. J. Hurst, S. H. Lewis, and R. G. Meyer, Analysis and design of analog integrated circuits, 5th ed. John Wiley and sons, 2010, ch. 8, p. 603.

[3] R. D. Middlebrook, "Null Double Injection And The Extra Element Theorem," IEEE Transactions On Education, vol. 32, no. 3, pp. 167180, AUG 1989.

[4] — "Low-entropy expressions: the key to design-oriented analysis," in Proceedings. Frontiers in Education. Twenty-First Annual Conference. Engineering Education in a New World Order. IEEE; American Soc. Eng. Educ, pp. 399-403.

[5] - The DT and the CT: The Dissection Theorem and the Chain Theorem. "Ch 09.DT \& CT.pdf". [Online]. Available: http://www.rdmiddlebrook.com/D_OA_Rules\&Tools/index.asp

[6] - "The two extra element theorem," in Proceedings. Frontiers in Education. Twenty-First Annual Conference. Engineering Education in a New World Order. IEEE; American Soc. Eng. Educ, pp. 702-708.

[7] F. Wiedmann. Loop gain simulation. [Online]. Available: http://sites.google.com/site/frankwiedmann/loopgain

[8] V. Vorpérian, Fast analytical techniques for electrical and electronic circuits. Cambridge, 2002.

[9] R. D. Middlebrook, V. Vorperian, and J. Lindal, "The N Extra Element Theorem," IEEE Transactions On Circuits And Systems I-Fundamental Theory And Applications, vol. 45, no. 9, pp. 919-935, SEP 1998.

[10] M. Tian, V. Visvanathan, J. Hantgan, and K. Kundert, "Striving for small-signal stability," IEEE Circuits \& Devices, vol. 17, no. 1, pp. 3141, JAN 2001. 